\title{
A NOTE ON LINK GROUPS
}

\author{
BY C. B. SCHAUFELE ${ }^{1}$ \\ Communicated by O. G. Harrold, September 15, 1965
}

1. Introduction. The purpose of this paper is to provide a sufficient geometric condition in order for a link group to be mapped homomorphically onto a free group of rank $r \leqq \mu$, where $\mu$ is the number of components of the link. Applying this together with a construction of L. Neuwirth [3], we obtain an extension of two results in [3] concerning free subgroups of link groups and solvability of link groups.

2. Group presentations. By a surface $S$ of type $(p, \mu, r)$ we shall mean the disjoint union in $S^{3}$ of $r$ tame, orientable, compact, connected 2-manifolds, each with nonempty boundary, where $\mu$ is the number of boundary components, and $p$ is the sum of the genera of the components of $S$. R. H. Fox describes in [1] a method of obtaining for any surface of type $(p, 1,1)$ a model $S$ of the same embedding type which consists of a 2-cell with a number of bands attached which may be made to "lie flat" so that only one side of $S$ is visible. This method may also be applied to a surface of type $(p, \mu, 1)$ and hence to a surface of type $(p, \mu, r)$, progressing componentwise.

If $L \subset S^{3}$ is a link with $\mu$ components and $S$ is a surface of type $(p, \mu, r)$ with boundary $L$, then a flat model of $S$ determines a regular projection of $L$ from which we obtain an over presentation [2]

$$
P_{L}=\left(x_{1}, \cdots, x_{m}: r_{1}, \cdots, r_{m}\right)
$$

of $\pi_{1}\left(S^{3}-L\right)$. Also, we can get a presentation

$$
P_{S}=\left(z_{1}, \cdots, z_{q}: s_{1}, \cdots, s_{v}\right)
$$

of $\pi_{1}\left(S^{3}-S\right)$, where each $z_{i}$ circles a band just once, and the relators occur where the bands cross each other. Each $z_{i}$ has the form $x_{i_{1}} x_{i_{2}}^{-1}$.

3. Main results. Let $L \subset S^{3}$ be a link with $\mu$ components and genus $p_{0}, G=\pi_{1}\left(S^{3}-L\right), F_{r}=$ free group of rank $r$.

THEOREM 1. If $L$ bounds a surface $S$ of type $(p, \mu, r)$, then there is an epimorphism $\phi: G \rightarrow F_{r}$.

\footnotetext{
1 Presented to the Society, April 10,1965. The contents of this paper are part of the author's doctoral dissertation at Florida State University, 1964, under the direction of M. L. Curtis. This research was supported by the National Science Foundation under contract GP 626 .
} 


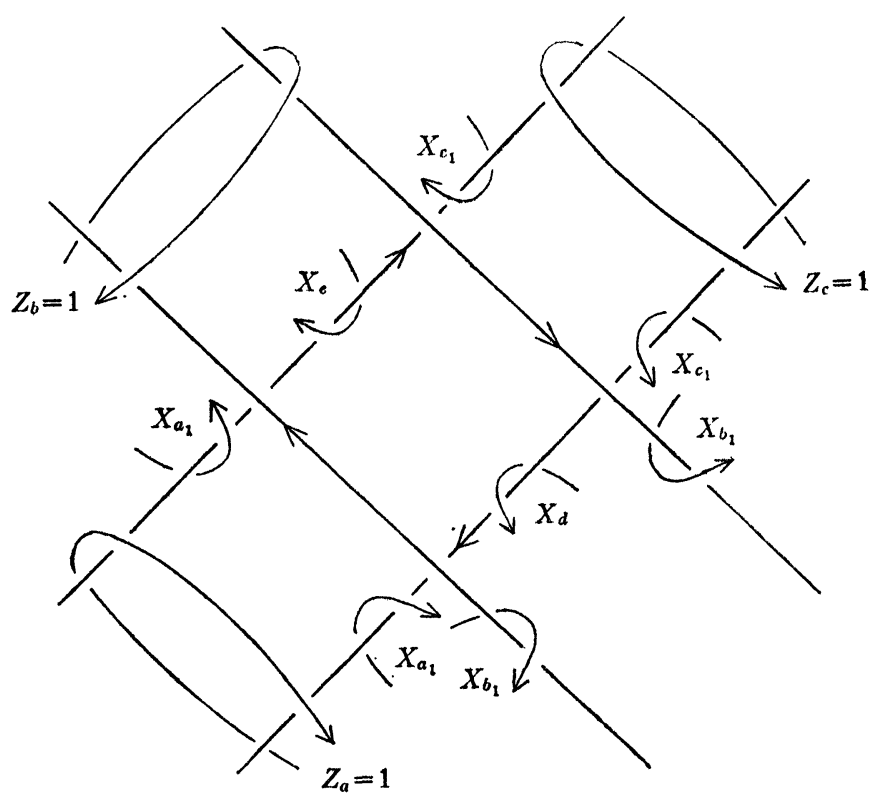

FIGURE 1.

Proof. Without loss of generality, we may assume that no component of $L$ is a trivial knot which is splittable from the rest of $L$. Let $H$ be the image of $\pi_{1}\left(S^{3}-S\right)$ in $G$ under the homomorphism induced by inclusion, and let $N(H)$ be the normal closure of $H$ in $G$. We have the presentations $P_{L}$ and $P_{S}$ given in $\$ 2$. A presentation $P$ of $G / N(H)$ can be obtained by adding $q$ relators $r_{1}^{\prime}=z_{1}, \cdots$, $r_{q}^{\prime}=z_{q}$ to $P_{L}$; thus the relations $r_{j}^{\prime}=1$ tell us $x_{j_{1}}=x_{j_{2}}$.

Consider first the case where $r=1$. In the regular projection of $L$ obtained from the flat model of $S$, the crossing points of $L$ occur where the bands of $S$ cross each other, so they occur in fours (see Figure 1).

By reading relations around these crossings, we obtain $x_{a_{1}}=x_{c_{1}}$ and $x_{d}=x_{e}$. Since adjacent bands are joined along the boundary of the 2-cell (Figure 2), it follows that $x_{a_{1}}=x_{b_{1}}=x_{c_{1}}$, so that $x_{a_{1}}=x_{d}$. Hence $x_{1}=x_{2}=\cdots=x_{m}$ and the relators $r_{i}$ become trivial since the sum of the exponents involved in each is zero. Thus $P$ is equivalent to the presentation $\left(x_{1}, \cdots, x_{m}: x_{1} x_{2}^{-1}, x_{2} x_{3}^{-1}, \cdots, x_{m-1} x_{m}^{-1}\right)$, so $G / N(H)$ $\approx F_{1}$.

Now suppose $r>1$. If no two bands which lie on different components of $S$ cross, then the above applies, componentwise, to give $G / N(H) \approx F_{r}$. 


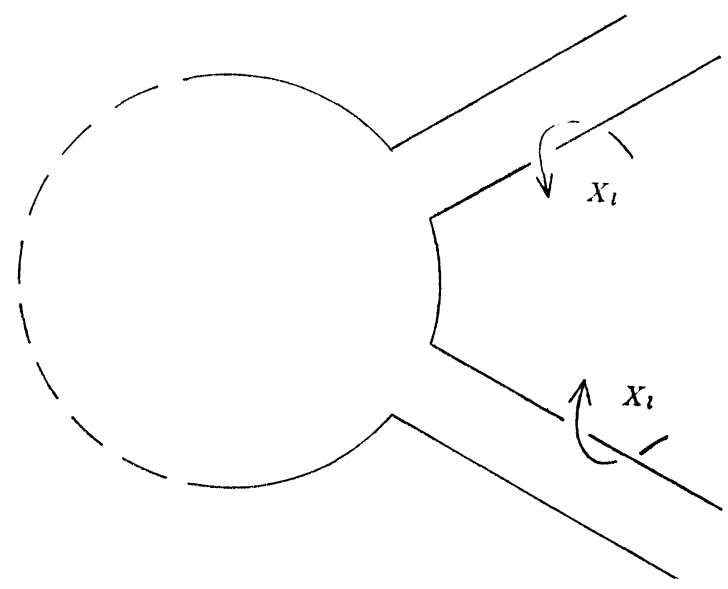

Figure 2.

Otherwise, consider Figure 1 where one band lies on one component of $S$, and the other band on another component. (At all crossings which involve only one component of $S$, we get all the $x_{i}$ 's around these four crossings equal as above, and so all the $r_{j}$ 's obtained at these four crossings become trivial.) We still get (as above) that $x_{a_{1}}=x_{c_{1}}$ and $x_{d}=x_{e}$. Now $x_{e}$ appears and only appears in the four relators obtained at the four crossings shown; these relators are the same, viz., $x_{e}^{-1} x_{b_{1}}^{-1} x_{a_{1}} x_{b_{1}}$. Hence $x_{e}=x_{b_{1}}^{-1} x_{a_{1}} x_{b_{1}}$ in $G / N(H)$, so this relator can be eliminated together with the generator $x_{e}=x_{d}$. It follows that $G / N(H) \approx F_{r}$.

THEOREM 2. If $p_{0} \geqq 1$ or if $p_{0}=0$ and $\mu>2$, then $G$ contains a free group of rank $n$ for any $n \leqq \infty$, and hence is not solvable.

Proof. It suffices to show that $G$ contains a free group of rank $\geqq 2$. If $L$ bounds a surface of type $(p, \mu, r)$ where $r \geqq 2$, then by Theorem 1 , $G$ can be mapped homomorphically onto $F_{r}$, and hence $G$ contains a free group of rank $r \geqq 2$.

Now suppose $L$ bounds no disconnected surface, and let $S$ be a surface of type $\left(p_{0}, \mu, 1\right)$ with boundary $L$. We may now construct the covering space of $S^{3}-L$ corresponding to $N(H)$ just as done in [3]. Thus $\pi_{1}(S) \subset G$; and

$$
\operatorname{rank} \pi_{1}(S)=\operatorname{rank} H_{1}(S)=1-\chi(S)=\mu+2 p_{0}-1 \geqq 2,
$$

where $\chi(S)$ is the Euler characteristic of $S$. Since $\pi_{1}(S)$ is free, the first part of the theorem follows. 
Since any subgroup of a solvable group is solvable, $G$ cannot be solvable.

\section{REFERENCES}

1. R. H. Fox, A quick trip through knot theory, Topology of 3-manifolds, PrenticeHall, Englewood Cliffs, N. J., 1962; pp. 120-167.

2. R. H. Fox and R. H. Crowell, Introduction to knot theory, Ginn, New York, 1963.

3. L. Neuwirth, The algebraic determination of the genus of knots, Amer. J. Math. 82 (1960), 791-798.

Florida State University

\section{THE ENUMERATION OF LABELED TREES BY DEGREES}

\section{BY JOHN RIORDAN}

Communicated by M. Kac, September 28, 1965

1. In [1] Cayley showed that the total number of (free) trees with labeled vertices $v_{1}, \cdots, v_{n}$ is $n^{n-2}$ by exhibiting a correspondence between them and the terms of $\left(v_{1}+\cdots+v_{n}\right)^{n-2} v_{1} v_{2} \cdots v_{n}$. This note shows that the correspondence determines the trees of given degree specification (the degree of a point is the number of lines incident to it; the degree specification is $\left(k_{1}, k_{2}, \ldots\right)$ with $k_{i}$ the number of points of degree $i)$. More precisely, if $T\left(n ; k_{1}, k_{2}, \ldots\right)$ is the number of labeled trees with degree specification $\left(k_{1}, k_{2}, \ldots\right)$ it will be shown that

$$
\begin{aligned}
T_{n}\left(x_{1}, x_{2}, \cdots\right) & =\sum T\left(n ; k_{1}, k_{2}, \cdots\right) x_{1}^{k_{1}} x_{2}^{k_{2}} \cdots \\
& =x_{1}^{n} Y_{n-2}\left(f x_{2} x_{1}^{-1}, \cdots, f x_{n-1} x_{1}^{-1}\right)
\end{aligned}
$$

with $f^{k} \equiv f_{k}=(n)_{k}=n(n-1) \cdots(n-k+1)$, and $Y_{n}$ the Bell multivariable polynomial.

2. In symmetric function notation Cayley's expression is $(1)^{n-2}\left(1^{n}\right)$ on $n$ variables. The multinomial theorem in symmetric function form $[2$, p. 43$]$ is

$(1)^{n}=\sum \frac{n !}{1 !^{k_{1}} \cdots n !^{k_{n}}}\left(1^{k_{1} 2^{k_{2}}} \cdots n^{k_{n}}\right), \quad k_{1}+2 k_{2}+\cdots+n k_{n}=n$.

Hence, on $n$ variables 